\title{
Weekend and off-hour effects on the incidence of cerebral palsy: contribution of consolidated perinatal care
}

\author{
Satoshi Toyokawa ${ }^{1,2^{*}}$ (D) Junichi Hasegawa ${ }^{2,3}$, Tsuyomu Ikenoue ${ }^{4}$, Yuri Asano ${ }^{2}$, Emi Jojima $^{2}$, Shoji Satoh ${ }^{5}$, \\ Tomoaki Ikeda ${ }^{6}$, Kiyotake Ichizuka ${ }^{7}$, Satoru Takeda ${ }^{8}$, Nanako Tamiya ${ }^{9}$, Akihito Nakai ${ }^{10}$, Keiya Fujimori ${ }^{11}$, \\ Tsugio Maeda ${ }^{12}$, Hideaki Masuzaki ${ }^{13}$, Hideaki Suzuki ${ }^{2}$ and Shigeru Ueda ${ }^{2}$
}

\begin{abstract}
Objective: This study estimated the effects of weekend and off-hour childbirth and the size of perinatal medical care center on the incidence of cerebral palsy.

Methods: The cases were all children with severe cerebral palsy born in Japan from 2009 to 2012 whose data were stored at the Japan Obstetric Compensation System for Cerebral Palsy database, a nationally representative database. The inclusion criteria were the following: neonates born between January 2009 and December 2012 who had a birth weight of at least $2000 \mathrm{~g}$ and gestational age of at least 33 weeks and who had severe disability resulting from cerebral palsy independent of congenital causes or factors during the neonatal period or thereafter. Study participants were restricted to singletons and controls without report of death, scheduled cesarean section, or ambulance transportation. The controls were newborns, randomly selected by year and type of delivery (normal spontaneous delivery without cesarean section and emergency cesarean section) using a 1:10 case to control ratio sampled from the nationwide Japan Society of Obstetrics and Gynecology database.

Results: A total of 90 cerebral palsy cases and 900 controls having normal spontaneous delivery without cesarean section were selected, as were 92 cerebral palsy cases and 920 controls with emergent cesarean section. A significantly higher risk for cerebral palsy was found among cases that underwent emergent cesarean section on weekends (odds ratio [OR] 1.72, 95\% confidence interval [CI] 1.06-2.81) and during the night shift (OR 2.29, 95\% Cl 1.30-4.02). No significant risk was found among normal spontaneous deliveries on weekends (OR 1.63, 95\% Cl 0.972.73) or during the quasi-night shift (OR $1.26,95 \% \mathrm{Cl} 0.70-2.27)$. Regional perinatal care centers showed significantly higher risk for cerebral palsy in both emergent cesarean section (OR 2.35, 95\% Cl 1.47-3.77) and normal spontaneous delivery (OR 2.92, 95\% Cl 1.76-4.84).
\end{abstract}

Conclusion: Labor on weekends, during the night shift, and at regional perinatal medical care centers was associated with significantly elevated risk for cerebral palsy in emergency cesarean section.

Keywords: Access to healthcare, Nightshift, Intensification, Concentration, Japan, Cerebral palsy

\footnotetext{
* Correspondence: t-satoshi@umin.ac.jp

'Department of Public Health, Graduate School of Medicine, The University

of Tokyo, 7-3-1 Hongo, Bunkyo-ku, Tokyo 113-0033, Japan

${ }^{2}$ Department of the Japan Obstetric Compensation System for Cerebral Palsy,

Japan Council for Quality Health Care, Tokyo, Japan

Full list of author information is available at the end of the article
}

(c) The Author(s). 2020 Open Access This article is licensed under a Creative Commons Attribution 4.0 International License, which permits use, sharing, adaptation, distribution and reproduction in any medium or format, as long as you give appropriate credit to the original author(s) and the source, provide a link to the Creative Commons licence, and indicate if changes were made. The images or other third party material in this article are included in the article's Creative Commons licence, unless indicated otherwise in a credit line to the material. If material is not included in the article's Creative Commons licence and your intended use is not permitted by statutory regulation or exceeds the permitted use, you will need to obtain permission directly from the copyright holder. To view a copy of this licence, visit http://creativecommons.org/licenses/by/4.0/. The Creative Commons Public Domain Dedication waiver (http://creativecommons.org/publicdomain/zero/1.0/) applies to the data made available in this article, unless otherwise stated in a credit line to the data. 


\section{Key message}

Labor on weekends, during the night shift, and at regional perinatal medical care center was associated with significantly elevated risk for cerebral palsy.

\section{Introduction}

Over the past two decades, a growing body of evidence has demonstrated an association between work outside of the Monday through Friday schedule and increased morbidity and mortality in hospital settings [1]. Most studies that have found worsened outcomes in patients admitted during the weekend and during off-hour shifts have evaluated patients requiring urgent or emergency intervention, with conditions such as stroke [2], myocardial infarction [3], traumatic brain injury [4], pulmonary embolism [5], gastrointestinal hemorrhage [6], pediatric surgery [7], and mortality in critical care admission [8]. In obstetrical and perinatal outcomes, several studies have indicated the unfavorable influence of weekend and night delivery on neonatal death [9-14], birth-related injury,[14, 15] asphyxia, [16] intraventricular hemorrhage, [11] neonatal encephalopathy [17], maternal complication [18], and perinatal complication [12, 14, 18, 19]. Recent studies have analyzed the impact of staff fatigue [20], lack of medical personnel [17, 18], high volume [21], and institutional characteristics (e.g., academic teaching hospital [22] or secondary/tertiary hospital [23, 24]) on weekend and off-hour outcomes. A report from Sweden found an increased risk of asphyxia in infants born at night [16]. This indicates a possible negative weekend and off-hour effect on obstetrical care. Definitive conclusions have not been reached in this area due to narrowly focused research settings and the range of disease and symptom outcomes assessed [11, 18, 21, 25-28].

This study focuses on cerebral palsy (CP) as an outcome to evaluate weekend and off-hour effects in Japanese settings. In Japanese obstetrical care, stillbirth outcomes have improved, but risk for $\mathrm{CP}$ remains among surviving infants [29]. One study that used data from one hospital in Japan reported that adverse neonatal outcomes among cesarean births increased during the night [30]. The number of obstetricians is decreasing in Japan [31], and access to obstetric care is being reduced, especially on weekends and during off-hours. To tackle this urgent and problematic shortage, perinatal medical care has been consolidated, and tertiary (comprehensive and regional) perinatal care centers have been established. This consolidation may modify weekend and off-hour effects. This study evaluated whether there are weekend and off-hour effects and examined the potential effects of the type of perinatal medical care center on the incidence of $\mathrm{CP}$, using nationwide Japanese databases.

\section{Methods \\ Study design and participants}

This was a retrospective case-control study that used data extracted from nationwide Japanese databases, namely, the Japan Obstetric Compensation System for Cerebral Palsy (JOCSC) and the Japan Society of Obstetrics and Gynecology (JSOG). The JOCSC is a government-supervised compensation system intended to provide prompt, no-fault compensation for children diagnosed with severe $\mathrm{CP}$ caused by trauma during labor and delivery and for their families, as well as to provide information that could help in prevention, the early resolution of disputes, and improving the quality of obstetric health care. All cases were approved for compensation by the JOCSC Operations Branch review board, which consists of obstetricians, pediatricians, midwives, and lawyers. Inclusion criteria for this study were the following: neonates born between January 2009 and December 2012, with a birth weight of at least 2000 grams and a gestational age of at least 33 weeks, and with a severe disability resulting from $\mathrm{CP}$ independent of congenital causes or factors during the neonatal period or thereafter. A comparable and compatible database period was used because the diagnosis of $\mathrm{CP}$ cases completed at 5 years old in JOCSC criteria and the data format of JSOG database was changed in 2013. In CP cases, disability is certified as having a first- or second-degree severity, according to the grade of disability definitions in the Welfare of Physically Disabled Persons Act [32], equivalent to grade three or severe in the gross motor function classification system. The study flow diagram is shown in Fig. 1.

Control group data were obtained from the JSOG perinatal database, an offline national and peerless birth registry that was established in 1974. The JSOG database contains attending physician-submitted annual data, in a standardized common format, for all pregnant women treated at the 253 comprehensive and regional perinatal care centers of the Perinatal Research Network of Japan [33]. Both types of care centers accept high-risk pregnant women and newborns with serious conditions, and both offer advanced perinatal care. Comprehensive perinatal care centers have more staff and equipment for special care and accept round-the-clock emergency referrals [34]. Data are stored with strict quality control implemented by the JSOG Perinatal Committee. The JSOG database, described in detail previously [35], is the largest obstetrics database in Japan, representing about $10 \%$ of the total number of births throughout the country each year. The JSOG database was used, despite its 
Cerebral Palsy cases approved by the Japan Obstetric

Compensation System for Cerebral Palsy (JOCSC) in 2009-2012

with no congenital anomaly or neonatal factors, birth weight $\geq 2000$

g, and gestation $\geq 33$ weeks $\quad \mathbf{N}=\mathbf{9 5 1}$

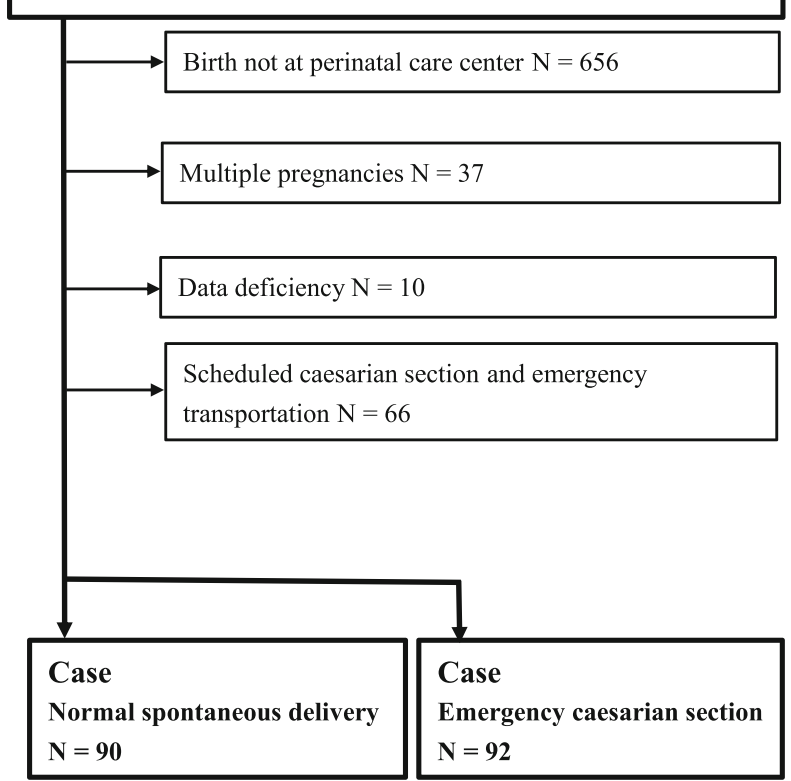

Fig. 1 Study flow diagram

lack of comprehensive hospital and clinic coverage, because no comparable database exists in Japan.

Generally, in case-control studies, case and control groups should be sampled from a common population in terms of causal CP relationships, dates, and times. Controls sampled from the JSOG included birth records from comprehensive and regional perinatal care centers to match the backgrounds of the cases. Births following transportation by ambulance from other facilities incorporate challenging backgrounds. Therefore, study participants were restricted to cases and controls from perinatal care center hospitals without ambulance transportation for whom complete data for all analyzed items were available in the JOCSC database (for those born 2009-2012) in March 2017 and in the JSOG database (for those born in 2009-2011). To avoid the inclusion of $\mathrm{CP}$ cases among the controls, controls who matched cases in terms of sex, date of birth, birth weight, gestational week, and maternal age were excluded. Cases and controls with scheduled cesarean sections were excluded because scheduled procedures typically do not occur on the weekend or during a night shift. Instrumental delivery was included in the normal (vaginal) spontaneous delivery category. Because obstetrical processes for normal spontaneous delivery and emergence cesarean were identical, we applied stratified matching and stratified analysis for normal spontaneous delivery and delivery
Controls from the perinatal database of the Japan Society of Obstetrics and Gynecology (JSOG) in 2009-2011 with no major congenital anomaly, born at a general or university hospital or perinatal center with birth weight $\geq 2000 \mathrm{~g}$ and gestation $\geq 33$ weeks $\mathbf{N}$ $=239,982$

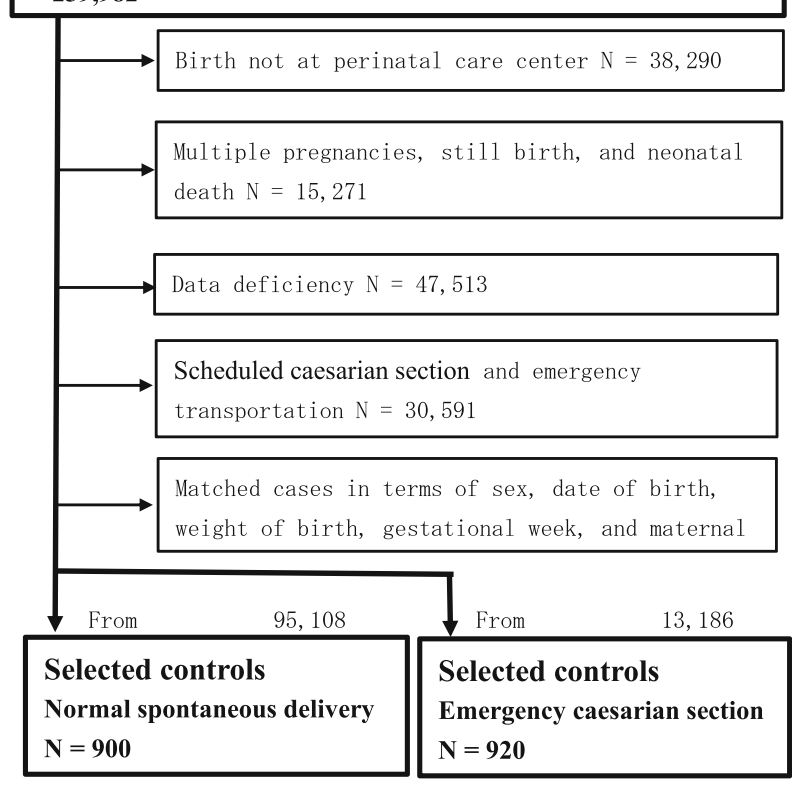

with emergent cesarean section. Matched analysis in matched sampled case-control studies can induce sampling bias [36], so we conducted un-matched analyses. Case and control data were extracted from the delivery medical records.

In case-control studies, matching is a means of providing a more efficient stratified analysis rather than a direct means of preventing confounding [36]. The data in the JSOG contained a few outlier values, typographical errors, and transfer errors from the hospital to the JSOG. To reduce the possibility of including inappropriate controls and to increase statistical power, cases were matched with 10 controls by birth year $(2009,2010$, and 2011-2012) and type of birth (normal spontaneous delivery without cesarean section and emergency cesarean section).

\section{Assessments}

The weekend was defined as Saturdays, Sundays, national holidays, and December 29-January 3. All other days were defined as weekdays. For the definition of offhour time shifts, we aggregated blocks of time into three shifts: day (0800-1800), quasi-night (1800-2400), and night (2400-0800). The definition of pregnancy-induced hypertension (PIH) included preeclampsia and gestational hypertension. Gestational hypertension was defined as onset of hypertension after 20 weeks' gestation 
(defined as systolic blood pressure of $140 \mathrm{mmHg}$ or diastolic blood pressure of $90 \mathrm{mmHg}$ ) on at least two occasions, $4 \mathrm{~h}$ apart. Preeclampsia was defined as gestational hypertension with proteinuria $(0.3 \mathrm{~g}$ in a 24 -h urine specimen or a protein-to-creatinine ratio of $>0.30$ ). Preterm labor is labor that begins before the 37th week of pregnancy. Fetal growth restriction was assessed using the standard deviation (SD) for birth weight [37].

\section{Statistical analyses}

Continuous variables were reported as means \pm SDs and were compared using Welch's $t$ test. Categorical variables were reported as frequencies and proportions and were compared using a chi-squared test. The relationships between CP and weekend/off-hour delivery were evaluated using multivariable logistic regression with $\mathrm{CP}$ as the dependent variable. The independent variables in Model 1 for cases and controls without cesarean section included day, perinatal care center level (comprehensive or regional ), shift, and year of delivery; Model 2 added the covariates prescription of prostaglandin or oxytocin, maternal age, parity, $\mathrm{PIH}$, preterm labor, gestational week, birth weight, and SD for birth weight. Models 3 and 4 included the same covariates as Models 1 and 2 for cases and controls with normal spontaneous delivery. Models 5 and 6 included the same covariates as Models 1 and 2 for cases and controls with emergency cesarean section. The results were expressed as an odds ratios (ORs) and 95\% confidence intervals (CIs). The goodness of fit of the multivariate models was evaluated using the Hosmer-Lemeshow test. Two-tailed $P$ values less than 0.05 were used to define statistical significance. All analyses were conducted using Stata version 13.0 (STATA Corporation, College Station, TX, USA).

\section{Results}

A total of 182 CP cases with normal spontaneous delivery without cesarean section and with emergent cesarean section (Table 1) were selected by year (2009, 64 [35.2\%]; 2010, 49 [26.9\%]; 2011-2012, 69 [37.9\%]) from the JOCSC database (Table 1). These were matched to 1820 controls, also sampled by year $(2009,640$ [35.2\%]; 2010, 490 [26.9\%]; 2011-2012, 690 [37.9\%]). In all cases of either normal spontaneous delivery or emergent cesarean section, significantly more preterm labor $(P<$ $0.001)$, fewer gestational weeks $(P<0.001)$, more fetus growth restriction (SD for birth weight; $P<0.001$ ), more weekend delivery $(P=0.015)$, and more use of regional perinatal care centers $(P<0.001)$ were seen in the $\mathrm{CP}$ cases.

Table 2 shows that significantly more cases than controls had night deliveries, adjusted for year and obstetric condition (Model 2: OR 1.56, CI 1.05-2.32). Significantly more cases than controls also had weekend deliveries,
Table 1 Demographics and prenatal participant characteristics

\begin{tabular}{|c|c|c|c|}
\hline Variable & $\mathrm{CP}(N=182)$ & Control $(N=1820)$ & $P$ value \\
\hline \multicolumn{4}{|l|}{ Birth year } \\
\hline 2009 & $64(35.2 \%)$ & $640(35.2 \%)$ & \multirow[t]{3}{*}{ NA } \\
\hline 2010 & 49 (26.9\%) & 490 (26.9\%) & \\
\hline 2011-2012 & 69 (37.9\%) & $690(37.9 \%)$ & \\
\hline \multicolumn{4}{|l|}{ Delivery } \\
\hline Normal & $90(49.5 \%)$ & $900(49.5 \%)$ & \multirow[t]{2}{*}{ NA } \\
\hline Emergency CS & $92(50.5 \%)$ & $920(50.5 \%)$ & \\
\hline Age, years & $32.0 \pm 5.1$ & $31.9 \pm 5.4$ & 0.865 \\
\hline \multicolumn{4}{|l|}{ Parity } \\
\hline One or more & 77 (42.3\%) & $688(37.8 \%)$ & $0.233^{\mathrm{a}}$ \\
\hline \multicolumn{4}{|l|}{ Prescription } \\
\hline Prostaglandin & $22(12.1 \%)$ & $156(8.6 \%)$ & $0.112^{\mathrm{a}}$ \\
\hline Oxytocin & $41(22.5 \%)$ & 479 (26.3\%) & $0.266^{a}$ \\
\hline $\mathrm{PIH}$ & $13(7.1 \%)$ & $116(6.4 \%)$ & $0.687^{a}$ \\
\hline Preterm labor & $68(37.4 \%)$ & $230(12.6 \%)$ & $<0.001^{\mathrm{a}}$ \\
\hline Gestational week & $37.8 \pm 2.3$ & $38.8 \pm 1.7$ & $<0.001$ \\
\hline SD for birth weight & $-0.304 \pm 1.032$ & $0.003 \pm 1.039$ & $<0.001$ \\
\hline \multicolumn{4}{|l|}{ Perinatal care centers } \\
\hline Comprehensive & $60(33.0 \%)$ & $992(54.5 \%)$ & \multirow[t]{2}{*}{$<0.001^{a}$} \\
\hline Regional & $122(67.0 \%)$ & $828(45.5 \%)$ & \\
\hline \multicolumn{4}{|l|}{ Day } \\
\hline Weekday & $122(67.0 \%)$ & $1344(73.9 \%)$ & \multirow[t]{2}{*}{$0.015^{\mathrm{a}}$} \\
\hline Weekend & 60 (33.0\%) & $476(26.2 \%)$ & \\
\hline \multicolumn{4}{|l|}{ Time shift } \\
\hline Day & 89 (48.9\%) & 1015 (55.4\%) & \multirow[t]{3}{*}{$0.088^{\mathrm{a}}$} \\
\hline Quasi-night & $44(24.2 \%)$ & 435 (23.9\%) & \\
\hline Night & 49 (26.9\%) & $370(20.3 \%)$ & \\
\hline
\end{tabular}

Data are expressed as means \pm SDs or numbers (percentages). $P$ values are derived from Welch's $t$ test

NA not applicable or being used in matching, CP cerebral palsy, $P I H$

pregnancy-induced hypertension, $C S$ caesarian section, $S D$ standard deviation

The chi-squared test

adjusted for year and obstetric condition (Model 2: OR 1.59 , CI 1.12-2.25). In addition, significantly more cases than controls had delivery in regional perinatal care centers, adjusted for year (Model 1: OR 2.41, CI 1.74-3.33) and obstetric condition (Model 2: OR 2.50, CI 1.78-3.50).

For those with normal spontaneous delivery (Table 3), significantly more cases than controls were delivered at regional perinatal care centers, adjusted for year (Model 3: OR 2.69, CI 1.69-4.30) and obstetric condition (Model 4: OR 2.92, CI 1.76-4.84).

For those with cesarean section (Table 4), significantly more cases than controls had weekend (OR 1.75, CI 1.09-2.80) or night (OR 2.40, CI 1.40-4.11) deliveries (Model 5). In the multivariable model adding obstetric conditions (Model 6), cases again had significantly more weekend (OR 1.72, CI 1.06-2.81) and night (OR 2.29, CI 
Table 2 Odds ratios for weekend and off-hour effects on cerebral palsy

\begin{tabular}{llll}
\hline Variable & Bivariate & Model 1 & Model 2 \\
\hline $\begin{array}{l}\text { Perinatal care centers } \\
\text { Comprehensive }\end{array}$ & 1.00 & 1.00 & 1.00 \\
$\begin{array}{l}\text { Regional } \\
\text { Day }\end{array}$ & $2.40,1.74-3.32$ & $2.41,1.74-3.33$ & $2.50,1.78-3.50$ \\
$\quad$ Weekday & 1.00 & 1.00 & 1.00 \\
$\quad$ Weekend & $1.50,1.08-2.08$ & $1.41,1.01-1.97$ & $1.59,1.12-2.25$ \\
Shift & & & \\
$\quad$ Day & 1.00 & 1.00 & 1.00 \\
$\quad$ Quasi-night & $1.25,0.86-1.83$ & $1.28,0.87-1.87$ & $1.29,0.87-1.92$ \\
$\quad$ Night & $1.48,1.02-2.14$ & $1.43,0.98-2.08$ & $1.56,1.05-2.32$ \\
Hosmer-Lemeshow test & & $P=0.636$ & $P=0.415$
\end{tabular}

Data are expressed as odds ratios and $95 \%$ confidence intervals with logistic regression models

Cases: $N=182$, controls: $N=1820$; covariates: (Model 1 ) birth year; (Model 2) adding birth year, prescription of prostaglandin, prescription of oxytocin, maternal age, parity, pregnancy-induced hypertension, preterm labor, gestational week, standard deviation for birth weight, and delivery

1.30-4.02) deliveries. Significantly more cases than controls had a cesarean section in a regional perinatal care center, adjusted for year (Model 5: OR 2.24, CI 1.423.53) and obstetric condition (Model 6: OR 2.35, CI 1.47-3.77).

The Hosmer-Lemeshow test showed a fairly good fit for all models (no significant $P$ values were observed in any models).

\section{Discussion}

Using two nationwide perinatal databases, our casecontrol study in comprehensive and regional perinatal

Table 3 Odds ratios for weekend and off-hour effects on cerebral palsy for normal spontaneous delivery

\begin{tabular}{llll}
\hline Variable & Bivariate & Model 1 & Model 2 \\
\hline $\begin{array}{l}\text { Perinatal care centers } \\
\text { Comprehensive }\end{array}$ & 1.00 & 1.00 & 1.00 \\
$\begin{array}{l}\text { Regional } \\
\text { Day }\end{array}$ & $2.65,1.66-4.21$ & $2.69,1.69-4.30$ & $2.92,1.76-4.84$ \\
Weekday & 1.00 & 1.00 & 1.00 \\
$\quad$ Weekend & $1.20,0.76-1.91$ & $1.17,0.73-1.88$ & $1.63,0.97-2.73$ \\
Shift & & & \\
Day & 1.00 & 1.00 & 1.00 \\
$\quad$ Quasi-night & $1.44,0.85-2.44$ & $1.51,0.89-2.58$ & $1.65,0.93-2.95$ \\
$\quad$ Night & $1.04,0.62-1.75$ & $1.00,0.59-1.71$ & $1.26,0.70-2.27$ \\
Hosmer-Lemeshow test & & $P=0.075$ & $P=0.897$ \\
\hline
\end{tabular}

Data are expressed as odds ratios and $95 \%$ confidence intervals with logistic regression models

Cases: $N=90$, controls: $N=900$; covariates: (Model 3) birth year; (Model 4) adding birth year, prescription of prostaglandin, prescription of oxytocin, maternal age, parity, pregnancy-induced hypertension, preterm labor, gestational week, and standard deviation for birth weight
Table 4 Odds ratios for weekend and off-hour effects on cerebral palsy for delivery with emergent caesarian section

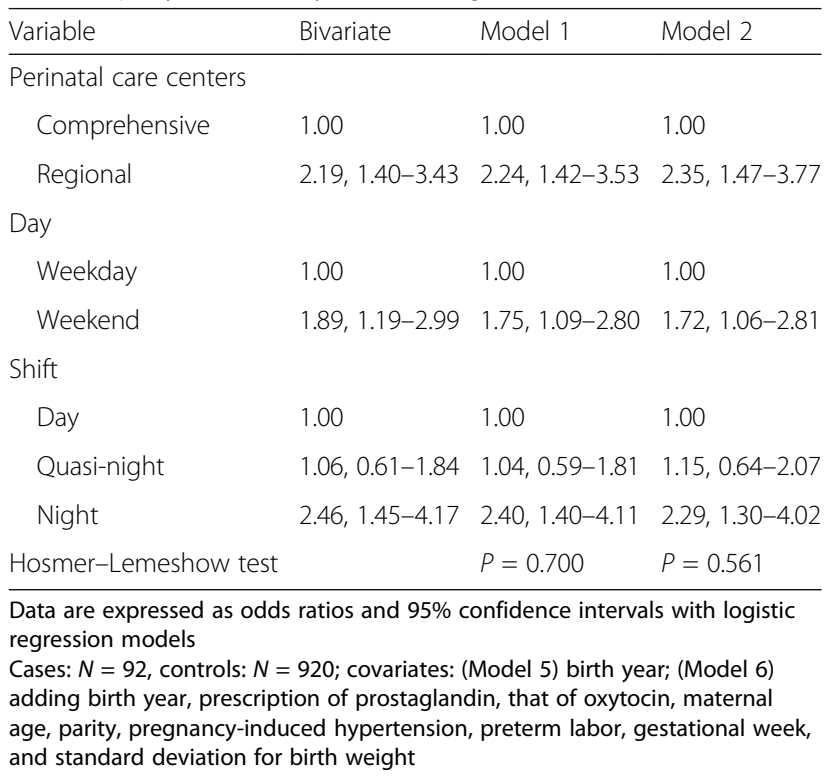

care centers found no weekend or off-hours effects on $\mathrm{CP}$ evident in normal spontaneous deliveries. However, we observed a 1.7 -fold weekend and 2.3-fold night on $\mathrm{CP}$ for delivery with emergency cesarean section without emergency transportation. To the best of our knowledge, this is the first study to investigate the risk for CP associated with delivery during the weekend and off-hour periods.

In Japan, approximately 2500 facilities provide delivery services for approximately one million deliveries per year [31]. Because pregnant women often prioritize the accessibility and comfort of delivery facilities, deliveries are not intensively performed, and more than half of all deliveries occur in private clinics operated by one or two obstetricians [31]. Nevertheless, the perinatal mortality rate and maternal mortality rate in Japan in 2012 were 4 per 1000 and 4 per 100,000 births, respectively [38], the lowest figures worldwide. Thus, the safety standards of prenatal practice in Japan are at least equivalent to those in other developed countries.

The incidence of CP in Japan is estimated to be 2.0 per 1000 births [39], which is about the same as that in other developed countries [40]. CP occurrence related to labor management should be limited, but the current study indicates that the risk might be elevated on the weekend and at night even in comprehensive and regional perinatal care centers that offer advanced perinatal care.

Previous studies on weekend and off-hour effects on obstetrical and perinatal outcomes have proposed several explanations: staff fatigue [20], a lack of medical personnel $[17,18]$, high volumes [21], and institutional characteristics [22-24]. Other previous studies have 
reported no difference in patient management on the weekend versus on weekdays for spontaneous labor [41] nor any increase in adverse maternal and neonatal outcomes from cesarean births at night [25]. Another study of weekend and off-hour effects on other health outcomes have offered explanations, such as fewer personnel, lower staffing levels, reduced numbers of senior staff, cross-cover of clinical specialties, proportionally more urgent procedures, deficits in continuity of care, and presenting patients often being in a more complex or more critical condition than those who typically present on weekdays [42]. Our target hospitals, comprehensive and regional perinatal care centers, must meet staff and facility requirements for $24 \mathrm{~h}$ operation; however, medical staff is reduced during the night and on weekends, meaning that fewer obstetricians are available to handle serious cases. Weekend and night $\mathrm{CP}$ effects during emergency cesarean sections might be the result of multiple simultaneous factors: lower staffing levels, fewer senior staff, cross-cover of clinical specialties, and proportionally more urgent procedures on weekends and at night.

In all models, regional perinatal care centers had a 2.2fold or higher risk of CP compared to comprehensive perinatal care centers. Generally, the minimum staffing and equipment requirements at regional perinatal care centers are lower than for comprehensive perinatal care centers [43]. Severe institutional risk conditions induced by deliveries may thus occur more frequently in regional perinatal care centers.

In a previous study, delays in diagnosis and treatment, including maternal transport, intervention, and blood transfusion, were associated with maternal death in Japan [26]. In fact, such cases encounter several problems in diagnostic procedures, treatment strategies, and inter- and intra-hospital systems [31]. In 2000, inadequate obstetric services were associated with maternal mortality in Japan. Reducing the number of deliveries in single-obstetrician facilities and establishing regional 24 $\mathrm{h}$ inpatient obstetric facilities for high-risk cases might reduce maternal mortality in Japan [44].

This weekend and holiday problem in regional perinatal care centers may reflect the current condition of obstetric medical care, in particular, the shortage of obstetricians. To tackle this shortage, the Medical Reform Committee of JSOG published its Grand Design 2015 [45], which recommended that obstetric services be consolidated in secondary and tertiary care hospitals, and regional and comprehensive perinatal care centers, to reach a sufficient number of full-time obstetricians. Consolidation of obstetric facilities impairs access, but a higher caseload and better staffing have the potential advantages of better clinical outcomes and reduced costs [46]. Metropolitan areas could develop plans to consolidate perinatal medical care; however, rural regions would not be able to achieve similar consolidation. The redistribution of care centers and obstetricians should also be accelerated. Comprehensive perinatal care centers are not distributed throughout the country to provide efficient access to perinatal care. According to the current results, appropriate management and intensive care is needed in regional perinatal care centers during the night and on weekends and holidays. Consolidation progressed during the study period 20092011. Further study is needed to fully capture the current situation.

This study has several strengths. First, we included a large number of $\mathrm{CP}$ patients from a nationwide database. Statistical power was estimated at 86.0, 62.6, and 97.8\%, respectively, for night, weekend, and regional perinatal care center effects for the bivariate analysis of delivery with emergent cesarean section. Second, data were drawn from available medical information and did not include self-administrated questionnaires, attenuating potential recall bias. However, this study also had several limitations. First, to provide grounds for comparison, it focused on cases and controls in comprehensive and regional perinatal care centers without ambulance transportation. Labor in general obstetrics clinics and hospitals with a limited number of obstetricians and midwives might be associated with exaggerated risk during weekend and off-hour delivery or in emergent cases with ambulance transportation. Second, only participants with births at 33 or more weeks were eligible to participate, so night and weekend effects were not studied for births outside of these restrictions. Third, the quality of the dataset was not balanced because the JOCSC data were manually confirmed by JOCSC investigators but the JSOG data were automatically transcribed from electronic patient records. Individual risk behaviors, i.e., alcohol drinking and drug use, were assessed with different questions between databases. No pair of comparable databases exists. Individual risk behaviors occur more often during weekends and at night and may therefore be a cause of weekend and night effects produced in this study [2]. Pregnant women usually avoid risk behaviors; however, the potentially confounding effects of individual behaviors were not analyzed in the current study. Fourth, the time between amniotic rupture and total delivery was not included in the analysis due to uncertain data recording.

\section{Conclusions}

In conclusion, in a case-control analysis, we found that weekend and night delivery were significantly associated with an elevated risk of CP in emergency cesarean sections without ambulance transportation in comprehensive and regional perinatal care centers. Regional perinatal care 
centers exhibited significantly higher risk for $\mathrm{CP}$ than comprehensive perinatal care centers. These findings should motivate health policymakers to consolidate perinatal medical care and to ensure that management and intensive care are appropriately applied in regional perinatal care centers during the night as well as on weekends and holidays.

\section{Abbreviations \\ Cl: Confidence interval; CP: Cerebral palsy; JOCSC: The Japan Obstetric Compensation System for Cerebral Palsy; JSOG: The Japan Society of Obstetrics and Gynecology; OR: Odds ratio; PIH: Pregnancy-induced hypertension; SD: Standard deviation}

\section{Acknowledgements}

We thank Mio Sano, Sana Ono, Nozomi Kobayashi, Miyuki Takeuchi, and Natsumi Tsuchiya (Prevention Recurrence Team, Japan Council for Quality Health Care) for their excellent support in data collection, organization, and statistical analysis. We thank ServiceScape and Patric Findler (https:// servicescape.com/) for editing a draft of this manuscript.

\section{Authors' contributions}

ST contributed to the concept and design of the study and conducted the statistical analyses. ST, JH, YA, and EJ had full access to all study data and were responsible for data integrity, and $\mathrm{ST}$ and $\mathrm{JH}$ were responsible for the accuracy of the data analyses. YA, EJ, and SS contributed to the application to acquire the data. ST, JH, TI, YA, EJ SS, TI, KI, ST, NT, AN, KF, TM, HM, HS, and SU were responsible for the interpretation of the results and drafting the manuscript, critically reviewing, agreeing to publish it and controlling the decision to publish. HS and SU also supervised the study and provided administrative, technical, and material support. The authors read and approved the final manuscript.

\section{Funding}

The authors received no specific funding for this work and have no personal, political, intellectual, or religious interests in this work.

\section{Availability of data and materials}

The data used to derive our conclusions are unsuitable for public deposition due to ethical restrictions imposed by the institutional ethics committee, as the data contain sensitive information on participants and facilities.

\section{Ethics approval and consent to participate}

The study protocol was approved by the Institutional Review Board of the JOCSC (26-01). The use of the JSOG database was permitted by the NonProfit Neonatal Research Network Japan Organization. Written informed patient consent was not obtained from study participants. However, patients were provided with an official flyer that contained an announcement of the implementation of a "case control study of cerebral palsy and the prevention of its recurrence." Although the analysis was retrospective, the data for the anonymized JOCSC and the JSOG databases were collected in a normal clinical setting, ensuring that patient confidentiality was protected. All patient records/information were anonymized and de-identified prior to analysis and no personal data were used in the present study.

\section{Consent for publication}

All authors meet the appropriate authorship criteria, and they have read the manuscript and agreed to its being submitted for publication.

\section{Competing interests}

The authors declare that they have no competing interests.

\section{Author details}

'Department of Public Health, Graduate School of Medicine, The University of Tokyo, 7-3-1 Hongo, Bunkyo-ku, Tokyo 113-0033, Japan. ${ }^{2}$ Department of the Japan Obstetric Compensation System for Cerebral Palsy, Japan Council for Quality Health Care, Tokyo, Japan. ${ }^{3}$ Department of Obstetrics and Gynecology, St. Marianna University School of Medicine, Kanagawa, Japan. ${ }^{4}$ University of Miyazaki, Miyazaki, Japan. ${ }^{5}$ Maternal and Perinatal Care Center,
Oita Prefectural Hospital, Oita, Japan. ${ }^{6}$ Department of Obstetrics and Gynecology, Mie University Graduate School of Medicine, Mie, Japan. ${ }^{7}$ Department of Obstetrics and Gynecology, Showa University Northern Yokohama Hospital, Kanagawa, Japan. ${ }^{8}$ Department of Obstetrics and Gynecology, Juntendo University, Tokyo, Japan. ${ }^{9}$ Department of Health Services Research, Faculty of Medicine, University of Tsukuba, Ibaraki, Japan. ${ }^{10}$ Department of Obstetrics and Gynecology, Nippon Medical School, Tokyo, Japan. "'Department of Obstetrics and Gynecology, Fukushima Medical University, Fukushima, Japan. ${ }^{12}$ Maeda Clinic, Shizuoka, Japan. ${ }^{13}$ Department of Obstetrics and Gynecology, University of Nagasaki, Nagasaki, Japan.

Received: 22 July 2020 Accepted: 27 August 2020

Published online: 10 September 2020

\section{References}

1. Myers RP, Kaplan GG, Shaheen AAM. The effect of weekend versus weekday admission on outcomes of esophageal variceal hemorrhage. Can J Gastroenterol. 2009;23:495-501.

2. Kamitani S, Nishimura K, Nakamura F, Nishimura K, Nakamura F, Kada A, et al. Consciousness level and off-hour admission affect discharge outcome of acute stroke patients: a J-ASPECT study. J Am Heart Assoc. 2014;3: e001059. https://doi.org/10.1161/JAHA.114.001059.

3. Sorita A, Ahmed A, Starr SR, Thompson KM, Reed DA, Prokop L, et al. Offhour presentation and outcomes in patients with acute ischemic stroke: a systematic review and meta-analysis. Eur J Intern Med. 2014;25:394-400. https://doi.org/10.1016/j.ejim.2014.03.012.

4. Schneider EB, Hirani SA, Hambridge HL, Haut ER, Carlini AR, Castillo RC, et al. Beating the weekend trend: increased mortality in older adult traumatic brain injury (TBI) patients admitted on weekends. J Surg Res. 2012;177:295300. https://doi.org/10.1016/j.jss.2012.06.022

5. Aujesky D, Jiménez D, Mor MK, Geng M, Fine MJ, Ibrahim SA. Weekend versus weekday admission and mortality after acute pulmonary embolism. Circulation. 2009:119:962-8.

6. Ananthakrishnan AN, McGinley EL, Saeian K. Outcomes of weekend admissions for upper gastrointestinal hemorrhage: a nationwide analysis. Clin Gastroentero Hepatol. 2009;7:296-302e291. https://doi.org/10.1016/j.cgh.2008.08.013.

7. Goldstein SD, Papandria DJ, Aboagye J, Salazar JH, Van Arendonk K, Al-Omar $K$, et al. The "weekend effect" in pediatric surgery - increased mortality for children undergoing urgent surgery during the weekend. J Pediatr Surg. 2014;49:1087-91. https://doi.org/10.1016/j.jpedsurg.2014.01.001.

8. Thomas CJ, Smith RP, Uzoigwe CE, Braybrooke JR. The weekend effect: short-term mortality following admission with a hip fracture. Bone Joint J 2014;96-B(3):373-8.

9. Luo ZC, Liu S, Wilkins R, Kramer MS. Fetal and infant health study group of the Canadian perinatal surveillance system. Risks of stillbirth and early neonatal death by day of week. CMAJ. 2004;170(3):337-41.

10. Pasupathy D, Wood AM, Pell JP, Fleming M, Smith GC. Time of birth and risk of neonatal death at term: retrospective cohort study. BMJ. 2010:341:C3498. https://doi.org/10.1136/bmj.c3498.

11. Jensen EA, Lorch SA. Association between off-peak hour birth and neonatal morbidity and mortality among very low birth weight infants. J Pediatr. 2017;186:41-48.e4. https://doi.org/10.1016/j.jpeds.2017.02.007.

12. Gijsen R, Hukkelhoven CW, Schipper CM, Ogbu UC, de Bruin-Kooistra M, Westert GP. Effects of hospital delivery during off-hours on perinatal outcome in several subgroups: a retrospective cohort study. BMC Pregnancy Childbirth. 2012;12:92. https://doi.org/10.1186/1471-2393-12-92.

13. Gould JB, Qin C, Chavez G. Time of birth and the risk of neonatal death. Obstet Gynecol. 2005;106(2):352-8.

14. Bendavid E, Kaganova Y, Needleman J, Gruenberg L, Weissman IS. Complication rates on weekends and weekdays in US hospitals. Am J Med. 2007;120(5):422-8.

15. Urato AC, Craigo SD, Chelmow D, O'Brien WF. The association between time of birth and fetal injury resulting in death. Am J Obstet Gynecol. 2006; 195(6):1521-6.

16. Berglund S, Grunewald C, Pettersson H, Cnattingius S. Risk factors for asphyxia associated with substandard care during labor. Acta Obstet Gynecol Scand. 2010;89(1):39-48. https://doi.org/10.3109/ 00016340903418751

17. Wu YW, Pham TN, Danielsen B, Towner D, Smith L, Johnston SC. Nighttime delivery and risk of neonatal encephalopathy. Am J Obstet Gynecol. 2011; 204(1):37.e1-6. https://doi.org/10.1016/j.ajog.2010.09.022. 
18. Snowden JM, Kozhimannil KB, Muoto I, Caughey AB, McConnell KJ. A 'busy day' effect on perinatal complications of delivery on weekends: a retrospective cohort study. BMJ Qual Saf. 2017;26(1):e1. https://doi.org/10. 1136/bmjas-2016-005257.

19. de Graaf JP, Ravelli AC, Visser GH, Hukkelhoven C, Tong WH, Bonsel GJ, et al. Increased adverse perinatal outcome of hospital delivery at night. BJOG. 2010;117(9):1098-107. https://doi.org/10.1111/j.1471-0528.2010.02611.x.

20. Heller G, Misselwitz B, Schmidt S. Early neonatal mortality, asphyxia related deaths, and timing of low risk births in Hesse, Germany, 1990-8: observational study. BMJ. 2000;321(7256):274-5.

21. Snowden JM, Darney BG, Cheng YW, McConnell KJ, Caughey AB. Systems factors in obstetric care - the role of daily obstetric volume. Obstet Gynecol. 2013;122(4):851-7.

22. Caughey $A B$, Urato AC, Lee KA, Thiet MP, Washington AE, Laros RK Jr. Time of delivery and neonatal morbidity and mortality. Am J Obstet Gynecol. 2008;199(5):496.e1-5. https://doi.org/10.1016/j.ajog.2008.03.046.

23. O'Donoghue K, Sheridan CP, O'Sullivan K, Greene RA, Higgins JR. Timing of birth related to obstetric practice and neonatal outcome. Ir Med J. 2008; 101(7):205-7.

24. Rautava L, Lehtonen $L$, Peltola $M$, Korvenranta $E$, Korvenranta $H$, Linna M, et al. PERFECT preterm infant study group. The effect of birth in secondaryor tertiary-level hospitals in Finland on mortality in very preterm infants: a birth-register study. Pediatrics. 2007;119(1):e257-63.

25. Bailit JL, Landon MB, Thom E, Rouse DJ, Spong CY, Varner MW, et al. National Institute of Child Health and Human Development maternal-fetal medicine units network. The MFMU cesarean registry: impact of time of day on cesarean complications. Am J Obstet Gynecol. 2006;195(4):1132-7.

26. Gould JB, Qin C, Marks AR, Chavez G. Neonatal mortality in weekend vs weekday births. JAMA. 2003;289(22):2958-62.

27. Bell EF, Hansen NI, Morriss FH Jr, Stoll BJ, Ambalavanan N, Gould JB, et al. Eunice Kennedy Shriver national institute of child health and human development neonatal research network. Impact of timing of birth and resident duty-hour restrictions on outcomes for small preterm infants. Pediatrics. 2010;126(2):222-31. https://doi.org/10.1542/peds.2010-0456.

28. Hamilton P, Restrepo E. Sociodemographic factors associated with weekend birth and increased risk of neonatal mortality. J Obstet Gynecol Neonatal Nurs. 2006;35(2):208-14.

29. Reddihough DS, Collins KJ. The epidemiology and causes of cerebral palsy. Aus J Physiotherapy. 2003:49:7-12.

30. Suzuki S, Nakai M, Hiraizumi Y, Satomi M. Time of delivery and perinatal outcome. J Nippon Med Sch. 2010;77(5):277-81.

31. Hasegawa J, Sekizawa A, Tanaka H, et al. Current status of pregnancy-related maternal mortality in Japan: a report from the maternal death exploratory Committee in Japan. BMJ Open. 2016;6:e010304. https://doi.org/10.1136/ bmjopen-2015-010304.

32. Hasegawa J, Sekizawa A, Tanaka H, Katsuragi S, Osato K, Murakoshi T, et al. Relevant obstetric factors for cerebral palsy: from the nationwide obstetric compensation system in Japan. PLoS One. 2016;11:e0148122. https://doi. org/10.1371/journal.pone.0148122.

33. Non-Profit Neonatal Research Network Japan Organization. Japan: Neonatal Research Network Database. 2006. http://plaza.umin.ac.jp/nrndata/indexe. htm. Accessed 26 July 2019.

34. Unno N. The perinatal care system in Japan. Jpn Med Assoc J. 2011;54:23440.

35. Matsuda Y, Hayashi K, Shiozaki A, Kawamichi Y, Satoh S, Saito S. Comparison of risk factors for placental abruption and placenta previa: case-cohort study. J Obstet Gynaecol Res. 2011;37:538-46. https://doi.org/10.1111/j.14470756.2010.01408.x

36. Rothman KJ, Greenland S, Lash TL ed. Modern Epidemiology, third ed. Lippincott Williams \& Wilkins, USA, 2008;175-9.

37. Hasegawa J, Arakawa K, Nakamura M, Matsuoka R, Ichizuka K, Katsufumi O, et al. Analysis of placental weight centiles is useful to estimate cause of fetal growth restriction. J Obstet Gynaecol Res. 2011;37:1658-65.

38. Kamiya K, editor. Maternal and child health statistics in Japan. Tokyo: Mothers' and Children's Health and Welfare Association; 2013.

39. Toyokawa S, Maeda E, Kobayashi Y. Estimation of the number of children with cerebral palsy using nationwide health insurance claims data in Japan. Dev Med Child Neurol. 2017;59:317-21. https://doi.org/10.1111/dmcn.13278.

40. Oskoui M, Coutinho F, Dykeman J, Jetté N, Pringsheim T. An update on the prevalence of cerebral palsy: a systematic review and meta-analysis. Dev Med Child Neurol. 2013;55:509-19. https://doi.org/10.1111/dmcn.12080.
41. Woods CFG, Manohar S, Lindow SW. Obstetric consultant weekend on-call shift patterns have no effect on the management of spontaneous labour in a large maternity hospital. J Obstet and Gynaecol. 2013;33:802-5. https:// doi.org/10.3109/01443615.2013.813918.

42. Crowley RW, Yeoh HK, Stukenborg GJ, Medel R, Kassell NF, Dumont AS. Influence of weekend hospital admission on short-term mortality after intracerebral hemorrhage. Stroke. 2009;40:2387-92. https://doi.org/10.1161/ STROKEAHA.108.546572.

43. Ishikawa M. Challenges faced by Japan's perinatal care service system: with focuses on the disparity between different medical districts and the intensity of medical resources. J Jpn Assoc Health Care Administrators. 2015; 9:39-45.

44. Nagaya K, Fetters MD, Ishikawa M, et al. Causes of maternal mortality in Japan. JAMA. 2000;283:2661-7.

45. Japan Society of Obstetrics and Gynecology. Grand design 2015 (GD2015) renovation of the obstetrics and gynecology healthcare system in Japan. Tokyo. 2015. http://shusanki.org/theme_page.html?id=282. Accessed July 26, 2019 (in Japanese).

46. Koike S, Matsumoto M, Ide H, Kashima S, Atarashi H, Yasunaga H. The effect of concentrating obstetrics services in fewer hospitals on patient access: a simulation. Int J Health Geogr. 2016;15:4. https://doi.org/10.1186/s12942016-0035-y.

\section{Publisher's Note}

Springer Nature remains neutral with regard to jurisdictional claims in published maps and institutional affiliations.
Ready to submit your research? Choose BMC and benefit from:

- fast, convenient online submission

- thorough peer review by experienced researchers in your field

- rapid publication on acceptance

- support for research data, including large and complex data types

- gold Open Access which fosters wider collaboration and increased citations

- maximum visibility for your research: over $100 \mathrm{M}$ website views per year

At BMC, research is always in progress.

Learn more biomedcentral.com/submissions 